University of Wollongong

Research Online

Faculty of Engineering and Information

Faculty of Engineering and Information

Sciences - Papers: Part A

Sciences

$1-1-2013$

\title{
An application of nonlinear feature extraction - a case study for low speed slewing bearing condition monitoring and prognosis
}

Wahyu Caesarendra

University of Wollongong,wc026@uowmail.edu.au

Prabuono Buyung Kosasih

University of Wollongong, buyung@uow.edu.au

A K. Tieu

University of Wollongong, ktieu@uow.edu.au

Craig Moodie

cam920@uow.edu.au

Follow this and additional works at: https://ro.uow.edu.au/eispapers

Part of the Engineering Commons, and the Science and Technology Studies Commons

Research Online is the open access institutional repository for the University of Wollongong. For further information contact the UOW Library: research-pubs@uow.edu.au 


\title{
An application of nonlinear feature extraction - a case study for low speed slewing bearing condition monitoring and prognosis
}

\begin{abstract}
This paper presents the application of four nonlinear methods of feature extraction in slewing bearing condition monitoring and prognosis: these are largest Lyapunov exponent, fractal dimension, correlation dimension, and approximate entropy methods. Although correlation dimension and approximate entropy methods have been used previously, the largest Lyapunov exponent and fractal dimension methods have not been used in vibration condition monitoring to date. The vibration data of the laboratory slewing bearing test-rig run at $1 \mathrm{rpm}$ was acquired daily from February to August 2007 (138 days). As time progressed, a more accurate observation of the alteration of bearing condition from normal to faulty was obtained using nonlinear features extraction. These findings suggest that these methods provide superior descriptive information about bearing condition than time-domain features extraction, such as root mean square (RMS), variance, skewness and kurtosis.
\end{abstract}

\section{Keywords}

feature, nonlinear, extraction, application, study, case, low, speed, prognosis, monitoring, condition, bearing, slewing

\section{Disciplines}

Engineering | Science and Technology Studies

\section{Publication Details}

Caesarendra, W., Kosasih, P., Tieu, A. K. \& Moodie, C. (2013). An application of nonlinear feature extraction - a case study for low speed slewing bearing condition monitoring and prognosis. 2013 IEEE/ASME International Conference on Advanced Intelligent Mechatronics (AIM) (pp. 1713-1718). United States: IEEE. 


\title{
An Application of Nonlinear Feature Extraction - A Case Study for Low Speed Slewing Bearing Condition Monitoring and Prognosis
}

\author{
Wahyu Caesarendra, Buyung Kosasih, Kiet Tieu and Craig A.S. Moodie
}

\begin{abstract}
This paper presents the application of four nonlinear methods of feature extraction in slewing bearing condition monitoring and prognosis: these are largest Lyapunov exponent, fractal dimension, correlation dimension, and approximate entropy methods. Although correlation dimension and approximate entropy methods have been used previously, the largest Lyapunov exponent and fractal dimension methods have not been used in vibration condition monitoring to date. The vibration data of the laboratory slewing bearing test-rig run at $1 \mathrm{rpm}$ was acquired daily from February to August 2007 (138 days). As time progressed, a more accurate observation of the alteration of bearing condition from normal to faulty was obtained using nonlinear features extraction. These findings suggest that these methods provide superior descriptive information about bearing condition than time-domain features extraction, such as root mean square (RMS), variance, skewness and kurtosis.
\end{abstract}

\section{INTRODUCTION}

The monitoring of slewing bearing condition requires the selection and extraction of features of vibration signals. Features of vibration signals are the key points in clustering, classification, pattern recognition, condition monitoring, fault diagnosis, and prognosis methods. From numerous existing features, the selection of the most appropriate features for studying the condition of slewing bearings is not a simple task. Two different approaches to feature extraction used in bearing condition monitoring can be identified in the literature [1], [14], [15]. Both approaches extract linear timedomain features, such as root mean square (RMS), variance, skewness, and kurtosis. Further, the condition of the bearing is already known, and the extraction of features is used to identify the bearing condition. Hence the use of features is solely to convince the occurrence of faulty condition. These two approaches are not appropriate for the current project on the condition monitoring on very low speed slewing bearing for several reasons.

The approach used in [1], [15] is not appropriate for this project because in this approach faulty signals are usually acquired from artificial bearing faults or seeded defect on inner race or outer bearing race. In this study, collecting the

* Research supported by University of Wollongong through University Postgraduate Award (UPA) and International Postgraduate Tuition Award (IPTA).

W. Caesarendra is with the Mechanical, Material and Mechatronics Engineering, University of Wollongong, Wollongong, NSW 2522 Australia (phone: $612+$ 4221-3555; fax: 61 2+ 4221-3477; e-mail: wc026@uowmail.edu.au).

B. Kosasih is with the Mechanical, Material and Mechatronics Engineering, University of Wollongong, Wollongong, NSW 2522 Australia (e-mail: buyung@uow.edu.au).

Professor K. Tieu is with the Mechanical, Material and Mechatronics Engineering, University of Wollongong, Wollongong, NSW 2522 Australia (e-mail: ktieu@uow.edu.au). different kinds of slewing bearing signals from corresponding different fault conditions would be extraordinarily difficult. It is difficult because: (1) the slewing bearing is expensive. Therefore we could not buy more than one and apply different fault condition on each bearing; and (2) an artificial fault is difficult to apply on the bearing due to the advanced technology required to dismantle the outer race and inner race from the whole bearing.

The approach used in [14] is not appropriate for this study because the features are usually extracted from a well-known faulty bearing signal, where the signal is easily identified by the naked eye. In our study, however, it is very difficult to distinguish between faulty bearing signal and normal signal because they similar. This is because when the bearing runs in very low rotational speed, the generated vibration signal is very weak. The limitations of the methods discussed above motivated us to prove that the existing linear time-domain features, such as root mean square (RMS), variance, skewness, and kurtosis, are less sensitive in feature extraction for slewing bearing, and other potential features need to be identified. In this paper, we propose the application of four nonlinear methods for feature extraction: largest Lyapunov exponent, fractal dimension, correlation dimension and approximate entropy.

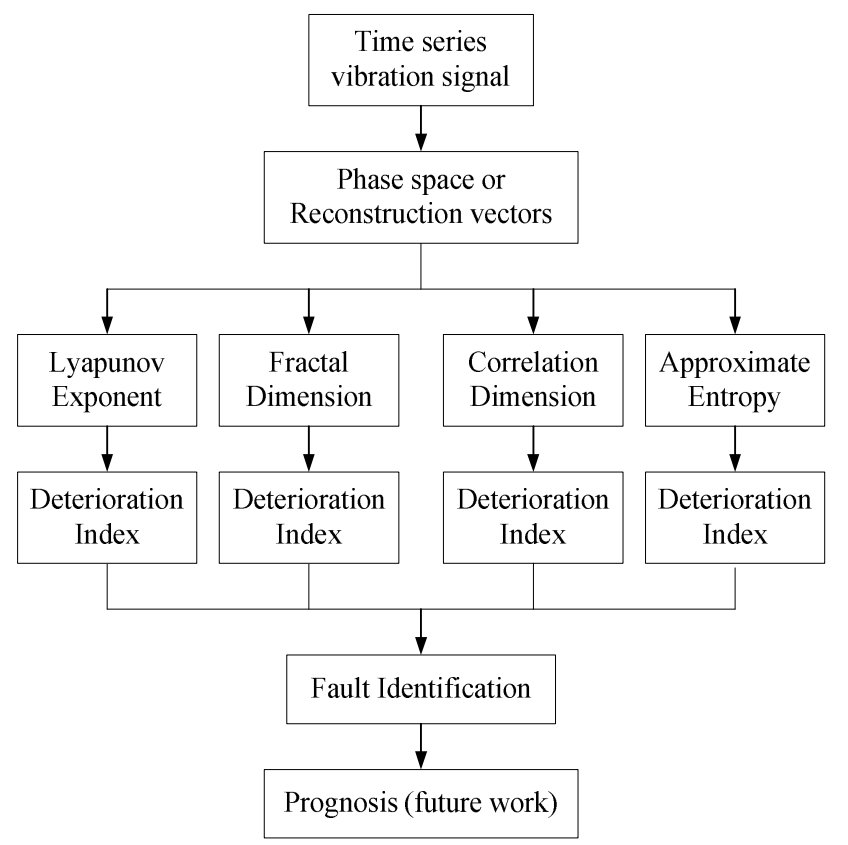

Figure 1. Flowchart of nonlinear features extraction application in slewing bearing condition monitoring and prognosis method. 


\section{NONLINEAR METHODS OF FEATURE EXTRACTION}

Slewing bearings are typically operating in very low rotational speed, in reversible mode, and under high axial and radial loads. When the slewing bearings start to deteriorate, the vibration signals cannot be treated as linear because the contacts between rolling elements and defects spots produce low energy local instabilities at irregular intervals. This low energy is deeply masked in background noise and susceptible to noise interference, consequently linear time-domain features fail to detect the faulty to normal bearing condition. In practice, multiple defects more frequently occur than single defects, therefore the vibration signals are considered completely irregular (chaotic) [2]. In this paper the application of four nonlinear methods of feature extraction: largest Lyapunov exponent, fractal dimension, correlation dimension and approximate entropy are presented. Unlike to linear features, nonlinear features are based on phase space or reconstruction vectors as shown in Fig. 1. Suppose $\mathbf{Y}=\left(y_{1}, y_{2}, \ldots, y_{N}\right)$ is the original time series or vibration signal in one second with sampling rate, $N$. The phase space or reconstruction vectors $\mathbf{X}$ of the original time series $\mathbf{Y}$ can be defined as follows:

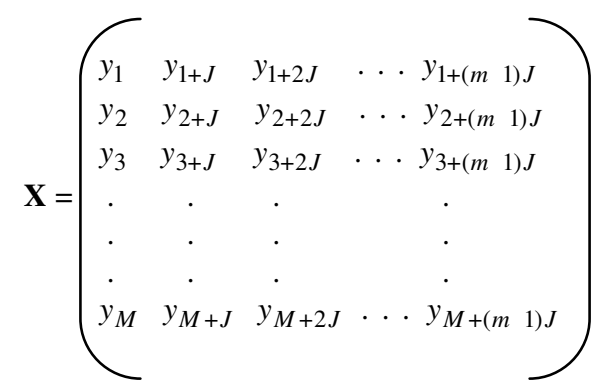

where $J$ represents reconstruction delay and can be computed by time lag/ $\Delta \mathrm{t}, m$ represents the embedding dimension and $M$ represents the number of reconstructed vectors. The relation between $N, J, m$ and $M$ can be defined in the following form: $N=M+(m-1) J$ or $M=N-(m-1) J$. Thus the dimension of $\mathbf{X}$ with predetermined $J$ and $m$ is an $M \times m$ matrix. For instance, if $\mathbf{Y}$ is the vibration data with $N=15, \mathbf{Y}=\left(y_{1}, y_{2}, \ldots, y_{15}\right)$, and select $J=2$ and $m=6$, thus the phase space of the vibration signal $\mathbf{Y}$ is the matrix $\mathbf{X}$ with size of $5 \times 6$ as defined:

$$
\mathbf{X}=\left(\begin{array}{llllll}
y_{1} & y_{3} & y_{5} & y_{7} & y_{9} & y_{11} \\
y_{2} & y_{4} & y_{6} & y_{8} & y_{10} & y_{12} \\
y_{3} & y_{5} & y_{7} & y_{9} & y_{11} & y_{13} \\
y_{4} & y_{6} & y_{8} & y_{10} & y_{12} & y_{14} \\
y_{5} & y_{7} & y_{9} & y_{11} & y_{13} & y_{15}
\end{array}\right)
$$

The nonlinear behavior of $\mathbf{X}$ in Eq. (1) or (1a) can be identified and analyzed using the nonlinear features extraction explained in subsections II.A through II.D.

\section{A. Largest Lyapunov exponent, $\lambda_{1}$}

The Lyapunov exponent algorithm is an old method and has been used in some areas such as biomedical engineering field especially in the analysis of electroencephalography (EEG) signal [3]. The Lyapunov exponent algorithm measures the exponential divergence (positive or negative) of two initial neighboring trajectories in a phase space. The objective is to quantify the appearance of disturbances corresponding to the signal abnormality. In other words, the Lyapunov exponent method measures the degree of chaos in certain time due to any disturbances. In this paper the term disturbances refers to any local instability vibration signal due to the dynamic contact between rolling elements and defect spots. This paper uses the largest Lyapunov exponent algorithm proposed by Rosenstein [4]. After the reconstruction vector in (1) computed, the largest Lyapunov exponent algorithm starts with the calculation of initial Euclidean distance, $\mathbf{d}_{\mathbf{0}}$ between phase space $\mathbf{X}$ and new phase space $\mathbf{X}_{\text {new }}$ as defined

$$
\mathbf{d}_{\mathbf{0}}(i)=\left\|\mathbf{X}-\mathbf{X}_{\mathbf{n e w}}(i)\right\|
$$

The new phase space $\mathbf{X}_{\text {new }}(i)$ is calculated by creating an $M \times m$ matrix, where the matrix of $\mathbf{X}_{\mathbf{n e w}}(i)$ for $i=1,2$, $\ldots, M$ is the row-vector replication of $\mathbf{X}(i)$. The additional constraint was determined to separate the nearest neighbors which are separated by a distance greater than the mean period of the vibration signal.

$$
|i-j|>\text { mean period }
$$

Introducing another counter as a sub looping to incorporate (3) in algorithm called $j$, where $j=1,2, \ldots, M$. Equation (3) means: if absolute value of subtraction $i^{\text {th }}$ and $j^{\text {th }}$ as $i$ and $j$ progresses is less than the mean period, the member of $\mathbf{d}_{\mathbf{0}}$ (with respect to $j$ ) is replaced by any a predetermined value which is much greater than the maximum value of $\mathbf{d}_{\mathbf{0}}(i)$. Thus the modified distance vectors, $\mathbf{d}_{\mathbf{0} \text { new }}(j)$ is obtained. The minimum distances $\boldsymbol{\Omega}_{\mathbf{0}}(i)$ and the position of minimum distances, $\boldsymbol{\Omega}_{\mathbf{m}}(i)$ of the modified distance vectors, $\mathbf{d}_{\mathbf{0} \_ \text {new }}(j)$ are then computed.

$$
\left[\boldsymbol{\Omega}_{\mathbf{0}}(i), \boldsymbol{\Omega}_{\mathbf{m}}(i)\right]=\min \left(\mathbf{d}_{\mathbf{0} \_ \text {new }}(j)\right)
$$

It noted that the value of initial minimum distance, $\boldsymbol{\Omega}_{\mathbf{0}}(i)$ is no used for further calculations. Only the position where the minimum distance is obtained denotes by $\boldsymbol{\Omega}_{\mathbf{m}}(i)$, is further employed.

The next step is the calculation of the new distance measure, $\mathbf{d}(i)$, which is measured the distance between $\mathbf{X}(i)$ and $\mathbf{X}\left(\boldsymbol{\Omega}_{\mathbf{p}}(i)\right)$ (with respect to $i$ ).

$$
\mathbf{d}(i)=\left\|\mathbf{X}-\mathbf{X}_{\text {new }}(\mathrm{i})\right\|
$$

In order to improve the accuracy of distance measures, $\mathbf{d}(i)$, Sato et. al [5] gives an alternative formula based on $k$ iteration. In this paper $k$ iteration is used from 1 , $2, \ldots, M$. Taking the sum of the $\mathbf{d}(i)$ and then dividing it by the number of point iterations, $M$, then compute the mean value of $\mathbf{d}(i)$ is computed; it becomes the modified distance measure, $\mathbf{d}_{\text {new }}(i)$ based on $k$ iteration. The formula is computed as

$$
\mathbf{d}_{\text {new }}(i)=\frac{1}{M} \sum_{i=1}^{M-k} \mathbf{d}(i)
$$

Referring to basic largest Lyapunov exponent formula [4], the relation between $\mathbf{d}_{\text {new }}(i)$ and largest Lyapunov exponent, $\lambda_{1}(i)$ defined by the following form: 


$$
\mathbf{d}_{\text {new }}(i)=C_{i} e^{\lambda_{1}(i . \Delta t)}
$$

where $C_{i}$ is the initial separation and $\Delta \mathrm{t}$ is the sampling period of vibration signal. By taking the logarithmic of both sides (7), a new formula is derived:

$$
\ln \mathbf{d}_{\text {new }}(i) \approx \ln C_{j}+\lambda_{1}(i . \Delta t)
$$

As seen in (8) that $C_{i}$ is unnecessary for estimating $\lambda_{1}$. Thus, the largest Lyapunov exponent can be computed using a least-square fitting equation defined by

$$
\lambda_{1}(i)=\frac{1}{\Delta t} \ln \mathbf{d}_{\text {new }}(i)
$$

\section{B. Fractal Dimension, $D$}

Fractal dimension can be used to measure the complexity of vibration signals. This method is one of phase space methods which examine the dynamical behaviours of phase space or reconstruction vectors. The commonly used algorithm of fractal dimension was proposed by Higuchi [6]. Once the phase space or signal reconstructed is obtained (1), the mean absolute length between the reconstructed vector $j^{\text {th }}$ and $\left(j^{\text {th }}-1\right)$ of $\mathbf{X}$ can be defined as follows:

$$
\begin{aligned}
& \text { for } i=1: J \\
& \text { for } j=2: m \\
& \qquad \begin{array}{l}
L_{1}(i, j-1)=|\mathbf{X}(i, j)-\mathbf{X}(i,(j-1))| \\
L_{m}(i, j-1)=\text { mean }\left(L_{1}\right)
\end{array} \\
& \text { end } \\
& \text { end }
\end{aligned}
$$

where $J$ represents reconstruction delay and $m$ is embedding dimension. Assuming that $L_{m}$ is proportional to $m^{-D}$, then taking the logarithmic natural of $(1 / m)$ and $L_{m}$. Hence, the fractal dimension, $D$ can be computed using standard leastsquare fitting method.

\section{Correlation Dimension, $C(l)$}

Correlation dimension provides a tool to quantify selfsimilarity. A larger correlation dimension corresponds to a larger degree of complexity and less-similarity. An earlier application of correlation dimension in bearing fault diagnosis was presented by Logan and Mathew [7]. The frequently used procedure to estimate the correlation dimension was introduced by Grassberger and Procaccia [8]. To calculate correlation dimension, the reconstructed matrix (1) are used as the input for the correlation dimension algorithm. The correlation dimension is derived from the correlation integral given by:

$$
C(l)=\lim _{M \rightarrow \infty} \frac{2}{M^{2}} \sum_{i=1}^{M-k} \sum_{j=i+k}^{M} \Theta\left(l\left|X_{i}-X_{j}\right|\right)
$$

where $X_{i}, X_{j}$ are the position vectors on attractor, $l$ is the distance under consideration, $\Theta(x)$ is the Heaviside step function, $\Theta(x)=0$ if $X \leqslant 0, \Theta(x)=1$ if $X>0, k$ is the summation offset, $M$ is the number of reconstructed vectors from the original vibration signal, and $C(l)$ is the correlation dimension.

\section{Approximate Entropy, ApEn}

The approximate entropy computes the value of regularity in the signal. Smaller value indicates more regular behaviour and higher value of it indicates less regularity on the data set. An earlier application of approximate entropy in bearing signal is presented by Yan, [9]; however, the paper discusses about the high speed rolling element bearing signal. Yan [9] mentioned that the deterioration of machine condition was followed by the increase of number of frequency components. This condition will decrease the regularity and increase the corresponding approximate entropy value. Similar to three other nonlinear features described above, approximate entropy uses reconstruction vectors (1) as an input. According to [9] the initial step of approximate entropy is measures the distance $d(X(i), X(j))$ between two vectors $X(i)$ and $X(j)$ which can be defined as the maximum difference in their respective corresponding elements:

$$
d(X(i), X(j))=\max _{k=1,2, \ldots, m}\left(\begin{array}{ll}
\mid x(i+k & 1) \\
x(j+k & 1) \mid)
\end{array}\right.
$$

where $i=1,2, \ldots, N-m+1, j=1,2, \ldots, N-m+1$, and $N$ is the number of data points contained in the time series. For each vector $X(i)$, a measure that describes the similarity between the vector $X(i)$ and all other vectors $X(j)$, where $j \neq i$ can be constructed as

$$
C_{i}^{m}(r)=\frac{1}{N-(m-1)} \sum_{j \neq i} \Theta\{r-d[X(i), X(j)]\}
$$

where $j=1,2, \ldots, N-m+1$ and the Heaviside step function, $\Theta(x)$ is similar to the symbol in correlation dimension where $\Theta(x)=0$ if $X \leqslant 0, \Theta(x)=1$ if $X>0$.

The symbol $r$ in (13) denotes a predetermined tolerance value, defined as

$$
r=k . s t d(\mathbf{Y})
$$

where $\operatorname{std}(\mathbf{Y})$ means is the standard deviation of original time series or vibration signal, $\mathbf{Y}$ and $k$ is a constant $(k>0)$. By defining

$$
\varphi^{m}(r)=\frac{1}{N-m+1} \sum_{i} \ln \left[C_{i}^{m}(r)\right], \quad i=1,2, \ldots, N \quad m+1
$$

the approximate entropy value of time series can be calculated as

$$
\operatorname{ApEn}(m, r)=\lim _{N \rightarrow \infty}\left[\varphi^{m}(r)-\varphi^{m+1}(r)\right]
$$

\section{SLEWING BEARING DATA}

The vibration accelerated life test data used in this paper were acquired from laboratory slewing bearing test-rig. The test-rig was operated in continuous rotation at speed of 1 $\mathrm{rpm}$. The slewing bearing used was an axial/radial bearing supplied by Schaeffler (INA YRT260) with an inner and outer diameter of $260 \mathrm{~mm}$ and $385 \mathrm{~mm}$. The vibration data were acquired from four accelerometers installed on the inner radial surface at 90 degree to each other. The accelerometers were IMI608 A11 ICP type sensor. The accelerometers were connected to high speed Pico scope DAQ (PS3424). To 
minimize noise from laboratory environment, the vibration data was collected at midnight outside of working hours with $4880 \mathrm{~Hz}$ sampling rates. The vibration data was collected during the period from February to August 2007 (138 days). In order to accelerate the bearing defect, coal dust was injected into the bearing on mid-April 2007 (58 days from beginning).

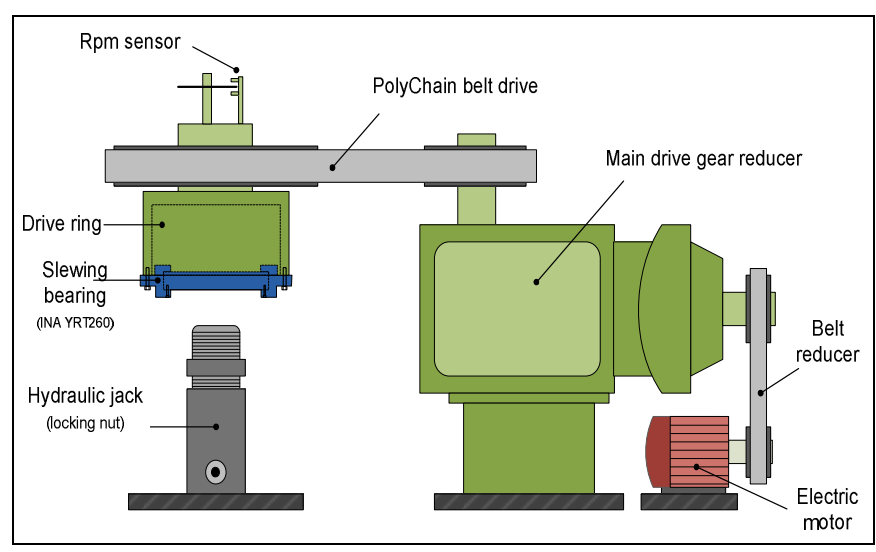

Figure 2. Schematic of laboratory slewing bearing test-rig.

The schematic of slewing bearing test-rig showing the main drive reducer, the hydraulic load and how the bearing is attached is shown in Fig. 2. The axial load was applied via hydraulic pressure approx. 30 tonnes.

\section{RESUlt AND DisCUSSION}

An initial vibration analysis was conducted to detect the fault occurrence in slewing bearing under test using FFT method. This method is very common in practice to see whether the fault has been occurred or not. The reference fault frequencies of slewing bearing are presented in Table I. If the dominant frequencies of FFT of slewing bearing data match with one or more of the fault frequencies shown in Table 1, it indicates fault has occurred. However, the energy spectrum of the slewing bearing is dominated by several high frequencies signal as shown in Fig. 3(b). The low energy slewing bearing signal is buried in high frequencies signal and make FFT fail to detect the bearing fault frequencies.

TABLE I. Slewing BEARING FAUlt FreQuencies [21]

\begin{tabular}{|c|c|c|}
\hline \multirow{2}{*}{ Defect Mode } & \multicolumn{2}{|c|}{ Fault frequencies (Hz) } \\
\cline { 2 - 3 } & Axial & Radial \\
\hline Outer ring (BPFI) & 1.32 & 0.55 \\
\hline Inner ring (BPFO) & 1.37 & 0.55 \\
\hline Rolling element (BSF) & 0.43 & 0.54 \\
\hline
\end{tabular}

The slewing bearing fault frequencies shown in Table 1 are calculated when the bearing rotates at $1 \mathrm{rpm}$. Different rotational speeds yield different fault frequencies.

Another condition monitoring method was carried out using time-domain features extraction such as root mean square (RMS), variance, skewness, and kurtosis. These features are commonly used due to the ease of computation and the certain physical sense. In high rotational speed bearing cases, these features are sensitive to represent the bearing condition. The increase of these features indicates the bearing condition is change. A brief description of those features and a list of references detailing some of their applications are presented in Table II.

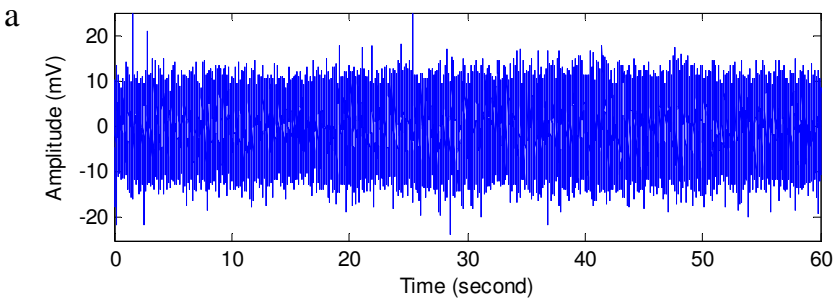

b

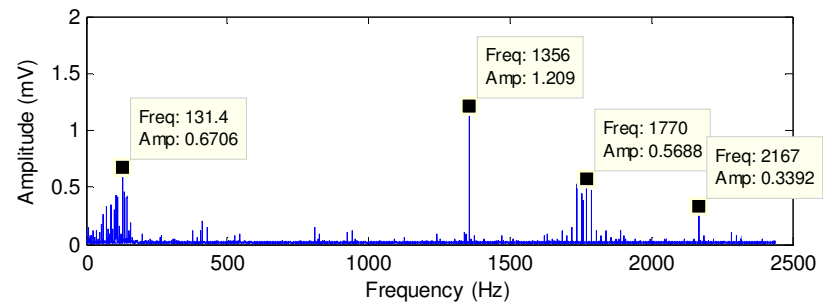

Figure 3. (a) Original vibration signal of $3^{\text {rd }}$ May (b) FFT.

The time-domain features extraction results of slewing bearing data are shown in Fig. 4. If the features are less sensitive, the increase of these features value related to bearing condition as time progresses is unable to detect, therefore the parameter called degradation index (DI) is needed. In this paper, DI is used to compare the performance of time-domain and nonlinear features extraction. It was simply calculated by measuring the fluctuation interval between maximum and minimum value of calculated feature respect to index of $i$. The DI is defined by

$$
\begin{aligned}
& \text { for } i=1: \mathrm{L} \\
& \qquad \mathrm{DI}(i)=\max (z(1: i))-\min (z(1: i)) \\
& \text { end }
\end{aligned}
$$

where, $\mathrm{L}$ is the number of data acquisition days from February to August, which is 138 in our case, and $z$ is the calculated feature which can be largest Lyapunov exponent feature, fractal dimension feature, correlation dimension feature or approximate entropy feature. The DI in (17) keeps the highest interval between maximum and minimum value of certain feature, $z$. It is updated once the current DI represents by $\mathrm{DI}(i)$ is greater than prior DI denotes by $\mathrm{DI}(i-1)$. This DI form is selected in this paper to facilitate the features with negative fluctuation as shown in RMS and kurtosis. In high rotational speed bearing cases, such thing is rarely occur, features are usually increased progressively when the bearing condition alter from normal to faulty.

Another aim of computed degradation index is for prediction purpose. In prognosis method DI is usually used as an object being predicted. The prognosis algorithm is expected to predict the posterior value of DI based on the prior values of DI. The common prognosis method used is 
data-driven method. The development of prognosis methods and algorithm based on calculated DI will be carried out in the future work.

TABLE II. TIME DOMAIN FEATURES

\begin{tabular}{|c|c|c|c|}
\hline \multirow{2}{*}{$\begin{array}{l}\text { Feature } \\
\text { Name }\end{array}$} & \multicolumn{3}{|c|}{ Description } \\
\hline & Brief Overview & Formula & Refs. \\
\hline$\sum_{2}^{\infty}$ & $\begin{array}{l}\text { As fault developed the } \\
\text { RMS value will } \\
\text { increase progressively. } \\
\text { However, RMS has } \\
\text { shortcoming that is } \\
\text { unable to provide the } \\
\text { information of incipient } \\
\text { fault stage while it } \\
\text { decreases with the fault } \\
\text { development [12]. }\end{array}$ & $\begin{array}{l}R M S= \\
\sqrt{\frac{\sum_{i=1}^{N} x_{i}^{2}}{N}}\end{array}$ & $\begin{array}{l}{[10],[11],} \\
{[12],[15]} \\
{[20]}\end{array}$ \\
\hline 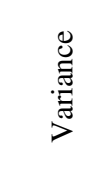 & $\begin{array}{l}\text { Variance quantifies the } \\
\text { dispersion of a signal or } \\
\text { a data set around their } \\
\text { mean reference. }\end{array}$ & $\begin{array}{l}\sigma^{2}= \\
\frac{\sum_{i=1}^{N}\left(x_{i}-m\right)^{2}}{(N-1) \sigma^{2}}\end{array}$ & $\begin{array}{l}\text { [13], [14], } \\
{[15],[16]}\end{array}$ \\
\hline 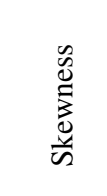 & $\begin{array}{l}\text { Skewness measures the } \\
\text { asymmetry of } \\
\text { probability density } \\
\text { function (pdf) of } \\
\text { vibration signal. }\end{array}$ & $\begin{array}{l}\gamma_{1}= \\
\frac{\sum_{i=1}^{N}\left(x_{i}-m\right)^{3}}{(N-1) \sigma^{3}}\end{array}$ & $\begin{array}{l}{[14],[15],} \\
{[16],[17],} \\
{[18]}\end{array}$ \\
\hline 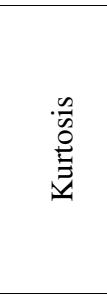 & $\begin{array}{l}\text { Kurtosis measures the } \\
\text { degree of flatness of the } \\
\text { probability density } \\
\text { function (pdf) near its } \\
\text { centre. In high speed } \\
\text { bearing rotation, the } \\
\text { kurtosis value of } \\
\text { bearing normal is well- } \\
\text { recognized as 3. }\end{array}$ & $\begin{array}{l}\beta_{2}= \\
\frac{\sum_{i=1}^{N}\left(x_{i}-m\right)^{4}}{(N-1) \sigma^{4}}\end{array}$ & $\begin{array}{l}{[12],[15],} \\
{[17],[19],} \\
{[20]}\end{array}$ \\
\hline
\end{tabular}

The DI of RMS, variance, skewness and kurtosis feature are presented in Fig. 5. According to Fig. 5 the increase of DI in time-domain features extraction result as day progresses is relatively small and it is not consistent among the four features. Thus the information related to bearing degradation condition especially initial severe failure is not clearly visible. The interesting results are shown in RMS and kurtosis which shows similar jumped DI in day 90.

Further, largest Lyapunov exponent, fractal dimension, correlation dimension and approximate entropy are extracted and the results are presented in Fig. 6. In contrast, the results show that there is a significant fluctuation in the last period of bearing operating time. The DI is also computed from these features and the results are shown in Fig. 7. Compared to time-domain features, the DI of the nonlinear features gradually increased and show consistent result. There is a maximum interval represents by $d$ occurred in four features in the day of 90 which similar to RMS and kurtosis features. This indicates the incipient severe failure. To clarify the assumption that there is a severe failure has occurred in slewing bearing, the slewing bearing was dismantled for inspection after August $31^{\text {st }} 2007$ which is after day 138. Considering the severity of the damage, it is expected that the damage had started around day 90 . Some of the defective regions can be clearly seen in Fig. 8 .
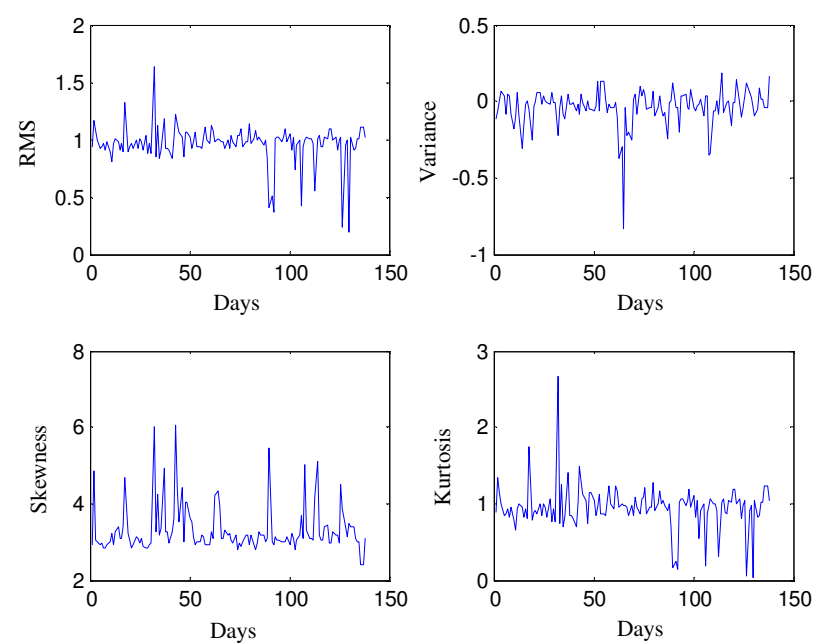

Figure 4. Time-domain features extraction results (138 days).
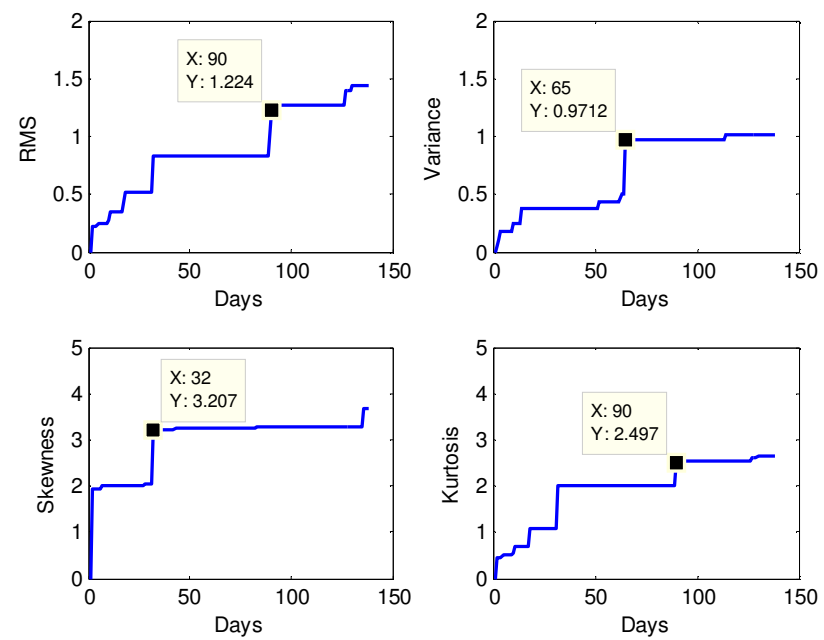

Figure 5. Deterioration index (DI) of time-domain features (138 days).
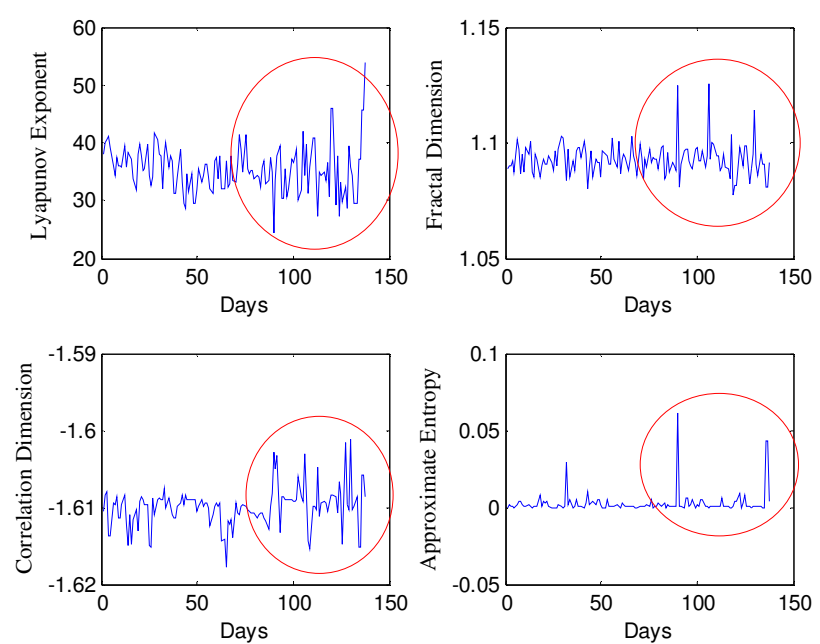

Figure 6. Nonlinear features extraction results (138 days). 

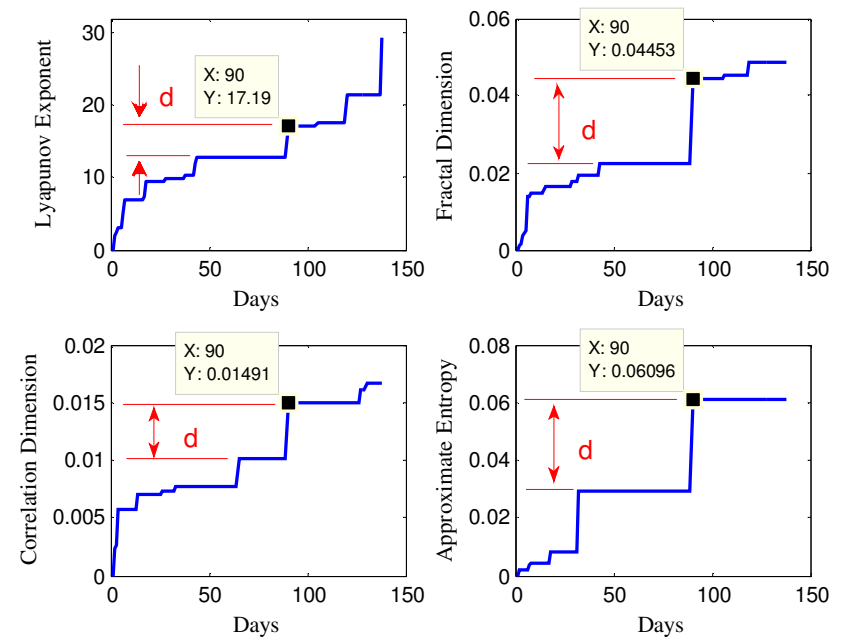

Figure 7. Deterioration index (DI) of nonlinear features (138 days).

(a)

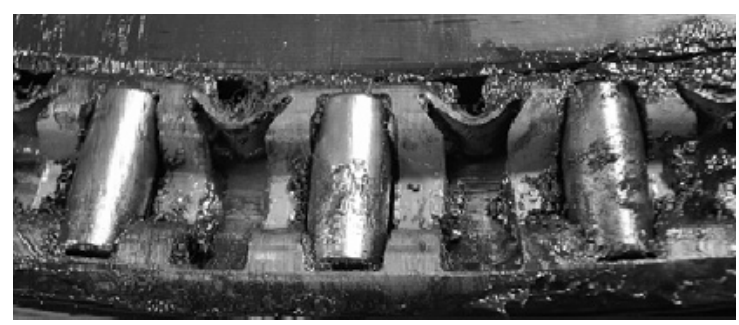

(b)

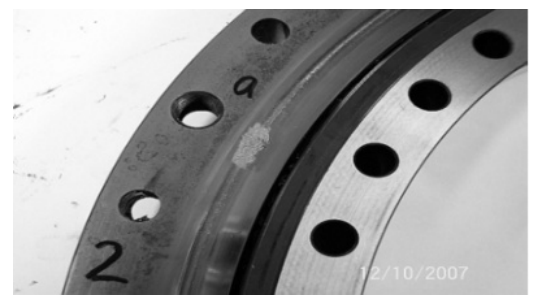

Figure 8. (a) A view of damaged rollers in axial plane (b) Outer raceway damage

\section{CONCLUSION}

The use of sensitive features for low-speed slewing bearing is an important factor in low-speed slewing bearing condition monitoring and prognosis. According to the selected features, deterioration index is computed. Monitored deterioration index is needed in order to set the threshold when the machine should be stopped or when the predictive maintenance should be carried out. By doing this the breakdown maintenance can be avoided. The merits of proposed nonlinear features have been explained. However, the shortcoming is the complexity of computation and the longer time their estimation takes than time-domain features.

\section{REFERENCES}

[1] A. Rojas and A. K. Nandi, "Detection and classification of rollingelement bearing fault using support vector machines," IEEE Workshop on Machine Learning for Signal Processing, Sept. 2005, pp. 153-158.
[2] L. M. Hively and V. Protopopescu, "Machine failure forewarning via phase-space dissimilarity measures, Chaos, Vol. 14, No. 2, pp. 408419, 2005.

[3] N. Paivinen, S. Lammi, A. Pitkanen, J. Nissinen, M. Penttonen, and T. Gronfors, "Epileptic seizure detection: A nonlinear viewpoint," Computer Methods and Programs in Biomedicine, Vol. 79, pp. 151159, 2005.

[4] M. T. Rosenstein, J. J. Collins, and C. J. De Luca, "A practical method for calculating largest Lyapunov exponents from small data sets," Physica D, Vol. 65, pp. 117-134, 1993.

[5] S. Sato, M. Sano, and Y. Sawada, "Practical methods of measuring the generalized dimension and the largest Lyapunov exponent in high dimensional chaotic systems," Progress of Theoretical Physics, Vol. 77 , no. $1,1987$.

[6] A. Accardo, M. Affinito, M. Carrozi, F. Bouquet, "Use of the fractal dimension for the analysis of electroencephalographic time series," Biological Cybernetics, Vol. 77, pp. 339-350, 1997.

[7] D. Logan and J. Mathew, "Using the correlation dimension for vibration fault diagnosis of rolling element bearings-I. Basics concepts," Mechanical Systems and Signal Processing, Vol. 10, No. 3, pp. 241-250, 1996.

[8] P. Grassberger and I. Procaccia, "Estimation of the Kolmogorov entropy from a chaotic signal," Physical Review, Vol. A28, pp. 25912593, 1983.

[9] R. Yan and R. X. Gao, "Approximate entropy as a diagnostic tool for machine health monitoring," Mechanical Systems and Signal Processing, Vol. 21, pp. 824-839, 2007.

[10] T. I. Liu and J. M. Mengel, "Intelligent monitoring of ball bearing conditions, " Mechanical Systems and Signal Processing, Vol. 6, No. 5, pp. 419-431, 1992.

[11] Y. Li, S. Billington, C. Zhang, T. Kurfess, S. Danyluk, and S. Liang, "Dynamic prognostic prediction of defect propagation on rolling element bearings," Tribology Transactions, Vol. 42, No. 2, pp. 385392, 1999.

[12] K. Shen, Z. He, X. Chen, C. Sun, and Z. Liu, "A monotonic degradation assessment index of rolling bearings using fuzzy support vector data description and running time," Sensor, Vol. 12, pp. 1010910135, 2012.

[13] S. Seker and E. Ayaz, "Feature extraction related to bearing damage in electric motors by wavelet analysis," Journal of The Franklin Institute, Vol. 340, pp. 125-134, 2003.

[14] V. Sugumaran, V. Muralidharan, and K. I. Ramachandran, "Feature selection using decision tree and classification through proximal support vector machine for fault diagnostics of roller bearing," Mechanical Systems and Signal Processing, Vol. 21, pp. 930-942, 2007.

[15] A. Widodo, E. Y. Kim, J. D. Son, B. S. Yang, A. C. C. Tan, D. S. Gu, B. K. Choi and J. Mathew, "Fault diagnosis of low speed bearing based on relevance vector machine and support vector machine," Expert Systems with Applications, Vol. 36, pp. 7252-7261, 2009.

[16] C. T. Yiakopoulus, K. C. Gryllias, and I. A. Antoniadis, "Rolling element bearing fault detection in industrial environments based on a $K$-means clustering approach," Expert Systems with Applications, Vol. 38, pp. 2888-2911, 2011.

[17] G. Niu, B. S. Yang, and M. Pecht, "Development of an optimized condition-based maintenance systems by data fusion and reliabilitycentered maintenance," Reliability Engineering and System Safety, Vol. 95, pp. 786-796, 2010.

[18] A. C. C. Tan, Y. -H. Kim, and V. Kosse, "Condition monitoring of low-speed bearing - a review," Australian Journal of Mechanical Engineering, Vol. 6, No. 1, pp. 61-68, 2008.

[19] W. Caesarendra, A. Widodo, and B. S. Yang, "Application of relevance vector machine and logistic regression for machine degradation assessment," Mechanical Systems and Signal Processing, Vol. 24, pp. 1161-1171, 2010.

[20] D. Siegel, H. Al-Atat, V. Shauche, L. Liao, J. Snyder, and J. Lee, "Novel method for rolling element bearing healt assessment - A tachometer-less synchronously average envelope feature extraction technique," Mechanical Systems and Signal Processing, Vol. 29, pp. 362-376.

[21] P. Eschmann, L. Hasbargen, and K. Weigand, "Die Wälzlagerpraxis: Handbuch für die Berechnung und Gestaltung von Lagerungen," Publisher: R. Oldenburg, 1953. 